\title{
Le projet d'agrandissement du lieu d'enfouissement sanitaire de Rimouski : quelques réflexions critiques
}

\author{
Chantal Quintin \\ Université du Québec à Rimouski
}

\section{Introduction}

$\mathcal{L}$ a plupart de nos biens de consommation aboutissent inévitablement sous une forme ou une autre à l'élimination et à l'enfouissement. Selon le portrait de la gestion des matières résiduelles de la région du Bas-Saint-Laurent ${ }^{1}$, cette dernière dispose de quatre modes de gestion dans les municipalités régionales de comté (MRC) : les lieux d'enfouissement sanitaire (LES), les dépôts de matériaux secs, les dépôts en tranchée et les lieux d'élimination ou de traitement des boues. Plus particulièrement, on trouve dans cette même région neuf lieux d'enfouissement sanitaire (tableau 1).

\section{Tableau 1 - Les lieux d'enfouissement sanitaire du Bas-Saint-Laurent}

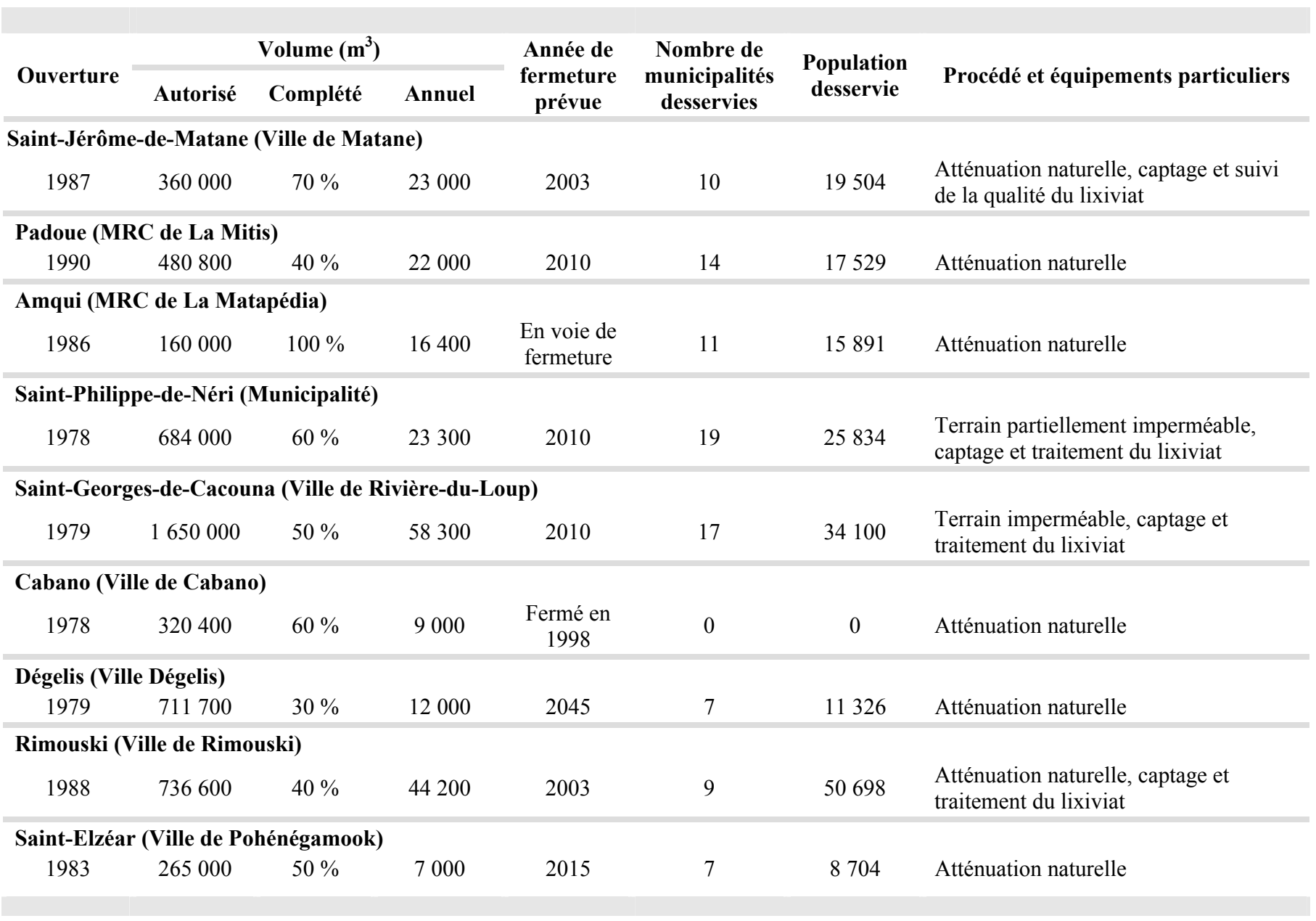

Source : Ministère de l'environnement, août 1998 (www.menv.gouv.qc.ca/matieres/mat_res/regions/bas-st-laurent.htm). 
Ce mode de gestion pour les déchets est la solution la plus utilisée actuellement, car elle est peu coûteuse. Toutefois, selon plusieurs sources, l'enfouissement sanitaire s'avère temporaire puisque les déchets sont accumulés et non éliminés. La majorité des déchets ne se décomposent pratiquement pas et demeurent intacts pendant plusieurs années. De plus, l'enfouissement de déchets engendre des eaux de lixiviation qui entraînent des polluants. Ceux-ci risquent de contaminer les cours d'eau et les nappes phréatiques.

L'objectif de cet article est d'identifier les impacts reliés au projet d'agrandissement du site d'enfouissement sanitaire de la ville de Rimouski à la fois aux plans environnemental, géographique et socio-économique dans une perspective de développement durable. De façon plus spécifique, il s'agit :

- d'identifier les éléments environnementaux défavorables à la localisation prévue pour l'implantation du lieu d'enfouissement technique (LET) de Rimouski;

- de préciser les coûts reliés à l'implantation du LET de Rimouski;

- d'identifier les éléments géographiques défavorables à la localisation prévue pour l'implantation du LET de Rimouski.

L'analyse a été réalisée à l'aide de photographies aériennes, de cartes topographiques d'échelles appropriées, de l'étude d'impacts sur l'environnement, de l'étude géotechnique et hydrologique effectuée par Technisol, une société de consultants en géotechnique et en contrôle des matériaux fondée en 1964. Nous avons également utilisé certains travaux portant sur la gestion des matières résiduelles ainsi que l'analyse sur l'évaluation du site d'enfouissement sanitaire de Rimouski ${ }^{2}$ réalisée par le géographe Clermont Dugas en 1979.

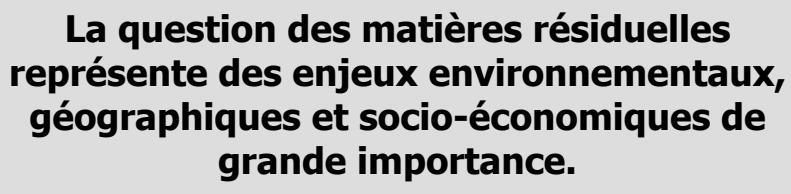

Dans de cette étude, nous retrouverons tout d'abord une mise en contexte suivie d'une description de l'aspect environnemental. Par la suite, nous présenterons une description des aspects géographique et socioéconomique de la localisation prévue pour l'implantation du LET de Rimouski. Finalement, nous décrirons des solutions envisageables orientées vers un développement durable ainsi que différents scénarios potentiels afin d'implanter un LET vers d'autres sites.

\section{Problématique}

\section{Mise en contexte}

En opération depuis 1981, le LES de Rimouski est situé sur le chemin Victor-Gauvin à environ six kilomètres au sud-ouest du centre-ville. La population actuellement desservie est de 50531 personnes provenant des municipalités de Saint-Anaclet-de-Lessard, de Saint-Fabien, de Saint-Valérien, du Bic et de Rimouski. Étant donné que sa capacité maximale d'enfouissement a été atteinte à l'été 2003, le projet d'agrandissement du LES actuel voit le jour. Ce projet vise l'aménagement d'un lieu d'enfouissement technique (LET) du coté sud-ouest du LES actuel, ayant une superficie de 22 ha et une durée de vie de 57 ans (carte 1). Contrairement a un LES, qui utilise un procédé par atténuation naturelle, un LET effectue la collecte et le traitement du lixiviat et du biogaz produits.

La question des matières résiduelles représente des enjeux environnementaux, géographiques et socioéconomiques de grande importance. Dans le cas où la méthode utilisée est l'enfouissement, les problèmes liés à la disposition des matières résiduelles ne font qu'augmenter. Cependant, une vision à long terme, par une saine gestion, permettrait de diminuer les conséquences pour différents aspects.

\section{Aspect environnemental}

Selon le guide d'implantation et de gestion de lieux d'enfouissement sécuritaire ${ }^{3}$, des travaux de nature géologique et hydrogéologique sont requis afin de juger la capacité d'un terrain à devenir un lieu d'enfouissement sécuritaire. On retrouvera alors ci-dessous certains résultats de l'étude géotechnique et hydrologique $^{4}$ démontrant que le terrain n'est pas favorable à l'enfouissement de matières résiduelles :

- la nature des matériaux de surface favorise, dans l'ensemble, un bon drainage du terrain sauf dans la 
portion sud où les sols cohérents favorisent le ruissellement de surface vers les dépressions de terrain ou les fossés périphériques du LES existant;

- la zone 2, située au sud et couvrant environ 16 hectares, se compose en surface d'une argile silteuse de consistance ferme à dure reposant sur le socle rocheux. L'épaisseur de l'argile varie entre 1,00 et plus de 4,90 mètres. Le socle rocheux atteint une profondeur variant entre 0,46 et 4,20 mètres;

- le degré de vulnérabilité de la nappe souterraine indique que le risque de contamination des eaux souterraines est considéré comme élevé, principalement en raison de matériaux perméables et de la proximité du niveau de la nappe d'eau souterraine.

\section{Carte 1 - Localisation du projet d'agrandissement du LES de Rimouski}

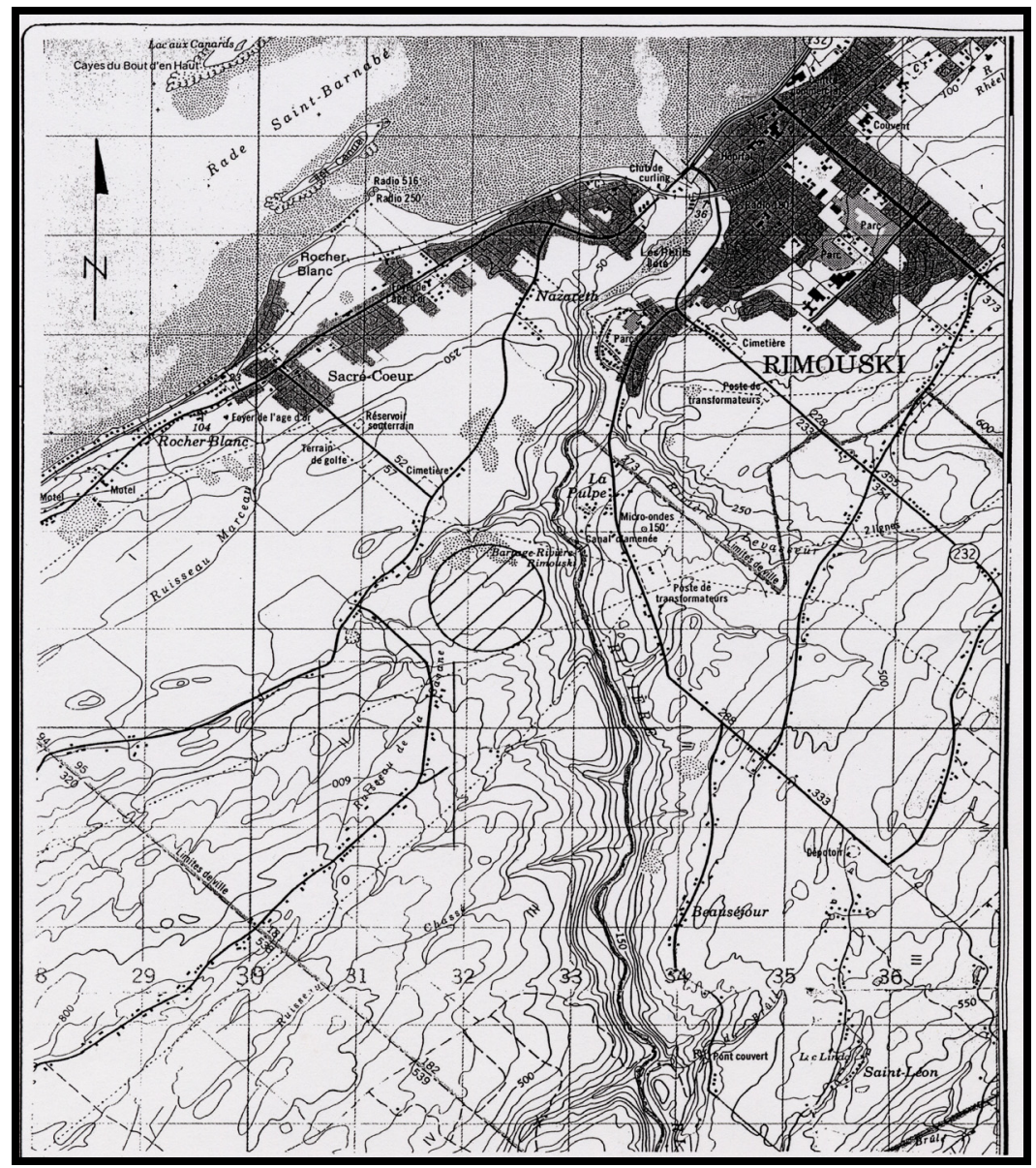

Source : Technisol inc., 2002.

Échelle : 1:50 000

D'après ces résultats, la localisation prévue pour l'implantation du LET est défavorable à l'enfouissement de matières résiduelles. En effet, le dépôt argileux a, à certains endroits, moins de trois mètres. Dans ce cas, le guide d'implantation et de gestion de lieux d'enfouissement sécuritaire indique qu'il faut utiliser un système de confinement à double géomembrane. Dans le cadre de ce projet d'implantation du LET, la géomembrane utilisée est en polythylène haute densité. Cette dernière a une durée de vie, dans des situations idéales, de plusieurs centaines d'années ${ }^{5}$. Cependant, sa durabilité dépend de plusieurs facteurs. Lors de l'installation, elle peut être abîmée par le transport et la manipulation ${ }^{6}$. Dans ce cas, son rôle, qui est de protéger la nappe phréatique de toute contamination, peut être remis en cause.

Afin de protéger l'environnement, l'enfouissement des déchets doit s'opérer selon les règles édictées par le Règlement sur les déchets solides. Le concept 
d'aménagement du LET de Rimouski est basé sur le projet de Règlement sur l'élimination des matières résiduelles annoncé par le ministre de l'Environnement le 25 octobre 2000. Ce nouveau cadre législatif prévoit remplacer le Règlement sur les déchets solides. Le projet d'aménagement respecte toutes les exigences de localisation prescrite par le règlement. Toutefois, certaines exigences de localisation par rapport à ce dernier sont toujours en vigueur. De plus, elles ne sont pas toutes respectées. Mentionnons notamment : «26. La distance de certains lieux: l'aire d'exploitation d'un lieu d'enfouissement sanitaire doit être située à plus de
150 mètres de tout parc municipal, terrain de golf, piste de ski alpin, base de plein air, plage publique, réserve écologique créée en vertu de la Loi sur les réserves écologique (L.R.Q., c. R-26.1), parc au sens de la Loi sur les parcs (L.R.Q., c. P-9), parc au sens de la Loi sur les parcs nationaux (Loi révisée du Canada (1985), chapitre N-14), mer, fleuve, rivière, ruisseau, étang, marécage ou batture (R.R.Q., 1981, c. Q-2, r. 14, a.26;L.Q., 1993, c.32, a.22)».

En effet, la carte hydrographique (carte 2) montre la présence d'un ruisseau intermittent à la limite sud de la sablière actuelle qui s'écoule vers l'ouest.

\section{Carte 2 - Carte hydrographique du LET de Rimouski}

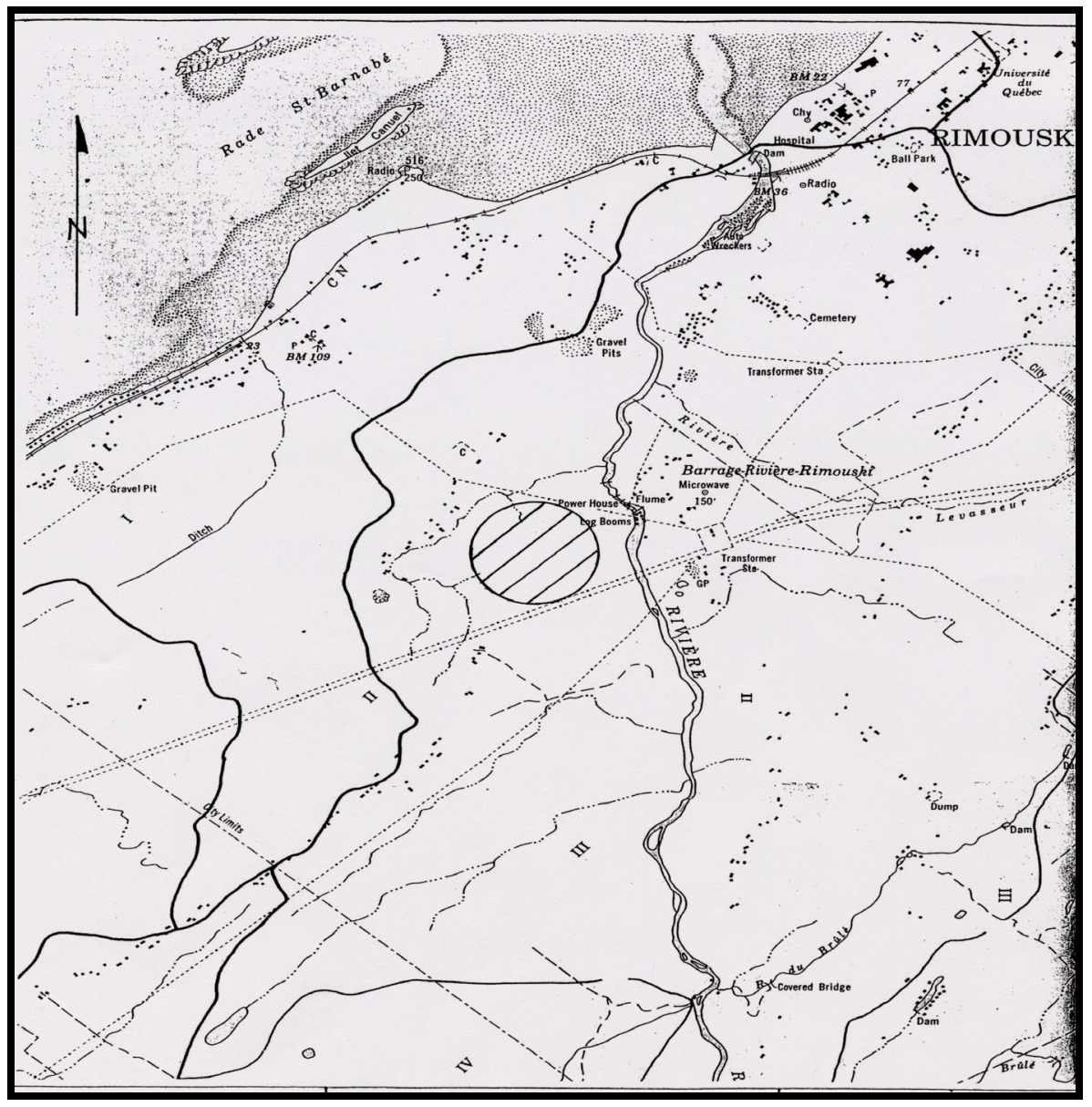

Source : Technisol inc., 2002.

Échelle : 1:50 000

L'enfouissement des déchets limite considérablement les possibilités d'utiliser le site dans le futur et ce, même durant plusieurs années après sa fermeture. La hausse des eaux de lixiviation et des biogaz produits aura pour conséquence d'augmenter les risques encourus par l'environnement et la santé. Selon le mi- 
nistère de l'Environnement, la matière putrescible est la principale cause de contamination dans les lieux d'élimination. À l'enfouissement, la fermentation en absence d'oxygène génère des gaz nauséabonds et explosifs qui contribuent à l'effet de serre. Les composés organiques libérés par cette fermentation migrent avec les eaux de lixiviation et peuvent contaminer tant les eaux de surface que les eaux souterraines et ainsi les rendre impropres à la consommation, en plus de constituer une menace pour la vie aquatique. Dans une approche de développement durable, l'en- fouissement de matières résiduelles est un mode de gestion de dernier recours.

\section{Aspect géographique}

D'après le schéma d'aménagement de la MRC de Rimouski-Neigette ${ }^{7}$, la localisation prévue pour l'implantation du LET de Rimouski comprend un site désigné comme un intérêt esthétique et naturel: les abords de la rivière Rimouski, et plus particulièrement La Pulpe (carte 3).

\section{Carte 3 - Activités récréotouristiques près du LET de Rimouski}

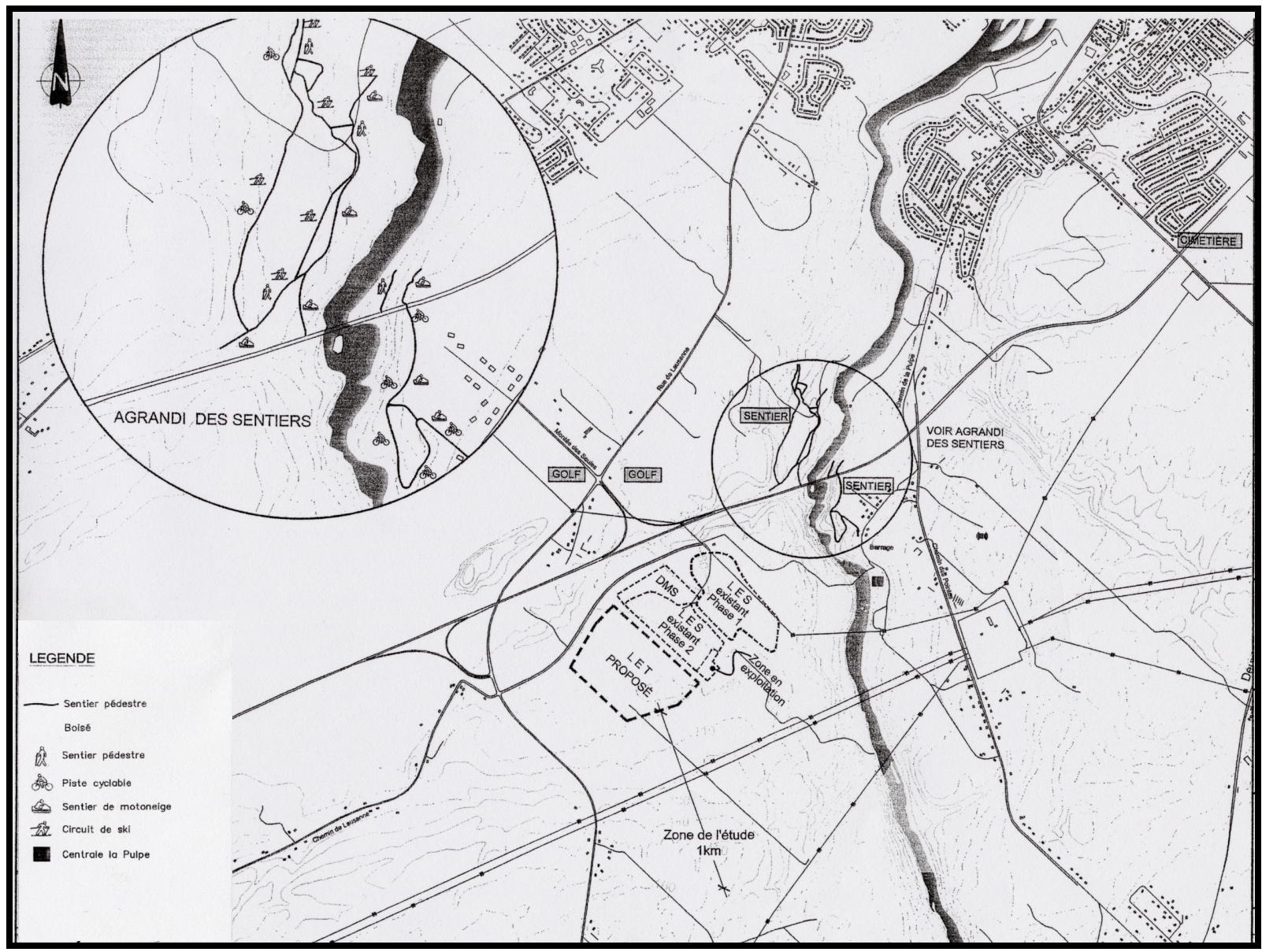

Source : Technisol inc., 2002.

Échelle : 1:20 000

Cette désignation signifie que ces sites, dont celui de La Pulpe, sont d'une beauté exceptionnelle et se trouvent à différents endroits sur le territoire de la MRC. Contrairement aux sites récréotouristiques, ces lieux sont aussi caractérisés par l'absence d'équipements de récréation. Toujours selon le schéma d'aménagement, l'intention de ce site est « de favoriser la conservation des caractéristiques esthétiques et naturelles, de conserver les caractéristiques physiques originales ainsi que d'éviter l'usage du site comme enfouissement des déchets ». De plus, La Pulpe a fait, l'objet, en 1986, d'un travail réalisé par l'Association des pêcheurs sportifs de saumons de la rivière Rimouski ${ }^{8}$ afin de déterminer son potentiel récréo- 
touristique. Ce travail a démontré que le site possédait, compte tenu de son histoire et de son milieu naturel, un potentiel pour réaliser des activités d'interprétation et de récréation comme la baignade, le kayak de rivière et la randonnée pédestre. La situation géographique actuelle du projet d'implantation $\mathrm{du}$ LET de Rimouski peut donc avoir un impact négatif (pollution par les eaux de lixiviation) en raison de la proximité d'un site comme celui de La Pulpe. Afin de le préserver, il est primordial de sauvegarder et de mettre en valeur ses qualités naturelles.

\section{Aspect socio-économique}

André Blouin, dans son Plan d'action pour une gestion intégrée des déchets ${ }^{9}$, sonne l'alarme en ce qui a trait aux coûts exorbitants nécessaires lorsque le LES actuel aura atteint sa capacité maximale d'enfouissement. « La fermeture du LES de Rimouski dans une dizaine d'années, soit en 2003, implique l'implantation d'un nouveau site qui engendrera des coûts fara- mineux à cause des études environnementales et des normes de plus en plus strictes imposées par le MENVIQ » mentionne-t-il. Pour ce qui est de l'implantation du LET de Rimouski, le coût total du projet représente 32,35 $\$ / \mathrm{t}$ comparativement à $27,17 \$ / \mathrm{t}$ pour celui de Matane (tableau 2).

Ce montant servira surtout à payer les infrastructures nécessaires à la conformation du site au projet de règlement sur les matières résiduelles. Ce règlement indique : "Afin d'empêcher la contamination du sol et des eaux souterraines par les lixiviats, les lieux d'enfouissement technique ne peuvent être aménagés que sur des terrains où les dépôts meubles sur lesquels seront déposées les matières résiduelles se composent d'une couche naturelle homogène ayant en permanence une conductivité hydraulique égale ou inférieure à $1 \times 10^{-6} \mathrm{~cm} / \mathrm{s}$ sur une épaisseur minimale de $6 \mathrm{~m}$, cette conductivité hydraulique devant être établie in situ $\gg{ }^{11}$.

\section{Tableau 2 - Synthèse des coûts d'élimination de Matane et de Rimouski ${ }^{10}$}

\begin{tabular}{lcc}
\hline Description & Rimouski & Matane \\
\hline Coût total d'aménagement du LET & $35712840 \$$ & $19916116 \$$ \\
Coûts d'opération & $638500 \$ / \mathrm{an}$ & 681000 \$/an \\
Contribution au fonds de poste-fermeture & $198500 \$ / \mathrm{an}$ & 272000 \$/an \\
Coût unitaire global a la tonne & $32.35 \$ / \mathrm{t}$ & $27.17 \$ / \mathrm{t}$ \\
\hline
\end{tabular}

Parce que le site n'est pas conforme à l'épaisseur minimale de six mètres, une bonne partie du montant est allouée à l'imperméabilisation du site.

L'aspect socio-économique englobe également les répercussions du projet pour la main-d'œuvre et l'achat de fournitures de biens et de services. La ville de Rimouski assurera la gestion et la coordination des activités au LET. Selon l'Étude d'impacts sur l'environnement, la composition et le nombre d'employés seront ajustés selon l'évolution du site. La plupart des emplois créés seront sur une base temporaire. Une perspective à long terme de la gestion de nos déchets et du choix du site serait rentable socio-économiquement. Elle permettrait ainsi de prolonger la durée de vie d'un lieu d'enfouissement, de créer des emplois permanents et d'assurer une meilleure qualité de vie aux générations futures.
Solutions envisageables

\section{Orientations vers un développement durable}

En septembre 2000, la Gazette officielle du Québec publiait la Politique québécoise sur la gestion des matières résiduelles 1998-2008 ${ }^{12}$. L'objectif général est de mettre en valeur, sur une base annuelle, plus de $65 \%$ de tonnes des 7,1 millions de matières résiduelles. Cette politique est basée sur des actions reposant sur cinq principes fondamentaux: la responsabilité élargie des producteurs, la participation des citoyens et des citoyennes, la régionalisation et le partenariat et le $3 R V-E^{13}$.

Comme cette politique le mentionne, les MRC doivent élaborer un programme de gestion des matières résiduelles. La MRC de Rimouski-Neigette est présentement en processus d'élaboration d'un tel plan. 
Selon le principe fondamental 3RV-E, il ressort qu'il est prématuré d'autoriser l'agrandissement du LES de Rimouski avant que ne soit déposé le plan de gestion des matières résiduelles de la MRC de RimouskiNeigette.

\section{Scénarios potentiels vers d'autres sites}

Selon l'étude d'impacts sur l'environnement, des scénarios potentiels afin d'implanter un LET sur le territoire de Rimouski-Neigette permettraient de trouver un emplacement naturellement imperméable tout en respectant les critères de localisation. Dans un rapport d'étude de levé géotechnique de la région de Rimous$\mathrm{ki}^{14}$, les résultats indiquent que l'épaisseur des dépôts meubles varie de quelques mètres à plus de 70 mètres. Plus particulièrement, les sédiments de la mer de Goldthwait forment l'unité la plus importante et la plus fréquemment rencontrée. Les auteurs affirment que l'épaisseur de cette unité oscille de quelques décimètres à plus de 65 mètres, notamment près du Bic. De plus, selon le mémoire réalisé par Jean-Sébastien Boucher $^{15}$, déposé au BAPE, il y aurait un site naturellement imperméable, situé à Pointe-au-Père, ayant entre 10 et 20 mètres d'argiles avant d'atteindre le roc.

Selon ces deux travaux, il ressort qu'il est nécessaire de procéder à l'élaboration d'une étude afin d'établir une liste de scénarios potentiels naturellement imperméables pour l'implantation d'un lieu d'enfouissement dans la MRC de Rimouski-Neigette.

\section{Conclusion}

Finalement, le projet d'agrandissement du lieu d'enfouissement sanitaire suscite beaucoup d'inquiétude chez les citoyens de Rimouski et des alentours. Cet article a permis de mettre en lumière des informations pertinentes sur le choix du site. En effet l'identification des impacts à la fois aux plans environnemental, géographique et socio-économique a permis de conclure que le choix du site actuel n'est pas le plus sécuritaire ni le moins coûteux. Nous reconnaissons que les activités humaines généreront toujours des matières résiduelles. Cependant, il existe des pratiques plus respectueuses de notre environnement. La solution passe tout d'abord par une saine gestion des matières résiduelles par le biais de la récupération, de la réduction, du recyclage et de la valorisation. Par la suite l'élimination doit se faire dans un endroit présentant des conditions favorables pour l'enfouissement de déchets. Ainsi, il sera possible d'économiser l'espace occupé dans les lieux d'enfouissement, ce qui prolongera la durée de vie du LES et restreindra le besoin d'en créer de nouveaux. Ce processus de gestion orientée vers un développement durable permettra de préserver notre qualité de vie et celle de nos enfants.

\section{Le projet d'agrandissement du lieu d'enfouissement sanitaire suscite beaucoup d'inquiétude chez les citoyens de Rimouski et des alentours.}

\section{Notes biographiques}

L'auteure est étudiante au baccalauréat en géographie à l'Univeristé du Québec à Rimouski. Elle tient à remercier Majella Simard, chercheur à la Chaire de recherche en développement rural, et Clermont Dugas, professeur de géographie, également à l'UQAR, pour leur collaboration à cet article.

\section{Notes et références}

1 Ministère de l'environnement (1998) : www.menv.gouv. qc.ca/matieres/mat_res/regions/bas-st-laurent.htm.

2 Dugas, C., G. Caron et C.-E. Bélanger (1979). Évaluation du site d'enfouissement sanitaire de Rimouski, UQAR, $41 \mathrm{p}$.

3 Gouvernement du Québec (1996). Lieux d'enfouissement sécuritaire: guide d'implantation et de gestion, SainteFoy, Québec, Publication du Québec.

4 Technisol (2002). Aménagement d'un lieu d'enfouissement technique à Rimouski : étude géotechnique et hydrogéologique, $23 \mathrm{p}$.

5 Giroud, J.P. et L.G. Tisinger (1993). " The Influence of Plasticizers on the Performance of PVC Geomembranes », Proceedings of Geosynthetic Liner Systems : Innovation, Concerns, and Design, IFAI, St. Paul, MN, p. 169-196.

6 Ministère de l'Environnement (1994). Les géomembranes en polyéthylène haute densité (PEHD), Gestion des sites d'enfouissement, p. 10-14 et 23-24.

7 MRC de Rimousiki-Neigette (adopté en mars 1987). Schéma d'aménagement; MRC de Rimouski-Neigette (22 sep- 
tembre 2003). Présentation relative au Schéma d'aménagement et aux orientations du Plan de gestion des matières résiduelles, non paginé.

8

Association des pêcheurs sportifs de saumons de la rivière Rimouski (1986). Potentiel recréo-touristique du site de la Pulpe, $31 \mathrm{p}$.

9

Blouin, A. (1992). Plan d'action pour une gestion intégrée Rimouski-Neigette : problématique et solutions régionales, MRC Rimouski-Neigette, Édition Rimouski, 125 p.

10

Simard André et al. (30 juillet 2002). Étude d'impacts sur l'environnement, déposée au ministre de l'Environnement, Volume 1 : Rapport principal, pagination diverse; Simard André et al. (30 juillet 2002). Étude d'impacts sur l'environnement, déposée au ministre de l'Environnement, Volume 2 : Annexes, pagination diverse; Simard André et al. (janvier 2003). Réponses aux questions et commentaires du ministère de l'Environnement, 37 pages et annexes; Simard, André et al. et SNC-LAVALIN (22 septembre
2003). Présentation du projet d'agrandissement du lieu d'enfouissement sanitaire de Rimouski, $17 \mathrm{p}$.

11 Ministère de l'environnement : http://www.menv.gouv. $\mathrm{qc} . c a /$ matieres/mat_res/consultation/chapitre1-2.htm.

12 Environnement Québec, Politique québécoise de gestion des matières résiduelles 1998-2008. http://www.menv. gouv.qc.ca/matieres/mat_res/parties1-4.htm\#avant-propos.

13 L'acronyme 3RV-E est défini comme la Réduction à la source, le Réemploi, le Recyclage, la Valorisation et l'Élimination. Ces dernières doivent être privilégiées dans cet ordre afin d'optimiser la gestion des matières résiduelles.

14 Dion D.-J. et R. Maranda (1978). Levé géotechnique de la région de Rimouski, rapport d'étude et carte d'aptitude, Ministère des Richesses naturelles, Direction de la géologie service de géotechnique, $31 \mathrm{p}$.

15 Boucher, J.-S. (2003). Projet d'agrandissement du lieu d'enfouissement sanitaire de Rimouski, $5 \mathrm{p}$. 